\title{
In vivo characterization of the psa genes from Streptococcus pneumoniae in multiple models of infection
}

Protein Design Labs, Inc., 34801 Campus Drive, Fremont, CA 94555, USA

\author{
Andrea Marra, $\uparrow$ Stacey Lawson, Jyoti S. Asundi, Daniel Brigham $\neq$ \\ and Alexander E. Hromockyj $\mathbb{S}$
}

Author for correspondence: Andrea Marra. Tel: +1 8606861507. Fax: +1 8604416159.
e-mail: andrea_marra@groton.pfizer.com

Keywords: manganese permease system, pneumococcal virulence

\section{INTRODUCTION}

In an attempt to identify novel targets for antimicrobial drug development, we have employed differential fluorescence induction (DFI) technology, which allows the identification of bacterial promoters expressed specifically in a given environment (Valdivia \& Falkow, 1996, 1997). This technology relies on the green fluorescent protein (GFP) reporter gene to indicate expression from a given promoter under different experimental conditions. In our application of this technology, random small fragments of chromosomal DNA from Streptococcus pneumoniae were cloned upstream of a promoterless gfp gene on an Escherichia coli-S. pneumoniae

\footnotetext{
+Present address: Pfizer Global Research and Development, Antibacterials Discovery MS8118W-249, Eastern Point Road, Groton, CT 06340, USA.

¥Present address: Genencor International, Inc., 925 Page Mill Road, Palo Alto, CA 94304, USA.

\Present address: Pharmacia Corporation, 7000 Portage Road, 6609209-737, Kalamazoo, MI 49001-0199, USA.

Abbreviations: DFI, differential fluorescence induction; FACS, fluorescence-activated cell sorting; GFP, green fluorescent protein; m.c.f., mean channel flourescence; RTI, respiratory tract infection; spc, spectinomycin.
}

shuttle plasmid (Bartilson et al., 2000). The resulting library was transformed into $S$. pneumoniae and grown under inducing and non-inducing conditions, and pools of clones grown under the inducing conditions were analysed and sorted by fluorescence-activated cell sorting (FACS). Subsequent rounds of growth followed by sorting resulted in a population enriched for clones containing promoters that are expressed under the inducing conditions.

S. pneumoniae is a Gram-positive bacterial pathogen responsible for causing serious community-acquired systemic (bacteraemia, meningitis) as well as more localized (upper respiratory tract, pneumonia, otitis media) infections (Kalin, 1998; Paton, 1996; Paton et al., 1993; Watson et al., 1995). Such infections are prevalent worldwide, and target especially infant, elderly and immunocompromised patients. One feature of S. pneumoniae pathogenesis that makes its infection so dangerous is its ability to disseminate and cause systemic infection that can progress to meningitis (Reynolds, 1999; Watson et al., 1995). This is in contrast to other causative agents of bacterial pneumonia, and as a result can lead to higher fatality rates for pneumococcal infections. 
Several important virulence determinants of this organism have been identified, yet little is known about the expression of these or other virulence factors during infection. In particular, the pneumococcal capsule has been shown to have a profound effect on virulence, with reports of $\sim 10^{6}$-fold reduction in virulence for capsule mutants (Kalin, 1998; Watson et al., 1995). The role of the capsule in the virulence of $S$. pneumoniae is not completely understood though it is likely to be important for in vivo survival by preventing phagocytosis of the organism by macrophages. Pneumolysin and autolysin have also been shown to be important for pathogenesis (Canvin et al., 1995; Paton, 1996), likely due to their involvement in cytotoxicity and quorum sensing, respectively. Mutants of $S$. pneumoniae lacking pneumolysin activity are unable to invade the bloodstream, suggesting that the cytotoxic effect of pneumolysin contributes to the invasive nature of pneumococcal infections (Canvin et al., 1995; Mitchell \& Andrew, 1997; Paton et al., 1997; Zysk et al., 2001). Potential pneumococcal vaccine candidates include PspA and PsaA, two surface proteins that are major immunogens (Brooks-Walter et al., 1999; Ogunniyi et al., 2000; Srivastava et al., 2000; Talkington et al., 1991, 1996).

We have previously used DFI to identify S. pneumoniae promoters induced under certain in vitro laboratory conditions (Bartilson et al., 2000; Marra et al., 2002) with the intention of mimicking aspects of the in vivo environment to which a pathogen may respond; subsequent experiments screened for promoters induced during infection. It was hoped that these identified promoters expressed genes whose functions were necessary for causing infection, and thus interruption of their expression would decrease virulence. The in vivo environment we chose for our screen was the murine lung during a $24 \mathrm{~h}$ infection.

Several rounds of infection followed by sorting by flow cytometry yielded a population of $S$. pneumoniae promoter-probe clones that have been collected on the basis of their high levels of expression during infection (Marra et al., 2002). Individual clones were isolated and the DNA inserts upstream of $g f p$ were sequenced. In one experiment, a disproportionate number of clones was found to carry the $p s a$ promoter indicating that the psaBCA operon is expressed during respiratory tract infection (RTI). The $p s a$ genes encode a manganese permease uptake system in S. pneumoniae (Dintilhac et al., 1997). psaB, psaC and $p s a A$ code for an ATPbinding protein, a transmembrane protein and a lipoprotein, respectively, and their identification has been reported previously (Dintilhac et al., 1997; Novak et al., 1998; Sampson et al., 1994). PsaA has strong homology to a number of Gram-positive bacterial surface proteins, namely AdcA from S. pneumoniae (Dintilhac \& Claverys, 1997), FimA from Streptococcus parasanguis (Burnette-Curley et al., 1995), ScaA from Streptococcus gordonii (Kolenbrander et al., 1998)and SsaB from Streptococcus sanguis (Ganeshkumar et al., 1991). psa mutants have been shown to require manganese for normal growth (Dintilhac et al., 1997) and to be attenuated in two murine models of $S$. pneumoniae infection (Berry \& Paton, 1996). We have extended these virulence studies and report here the analysis of $p s a$ expression in vitro and in vivo, and characterization of stable $p s a$ replacement mutants in four animal models of S. pneumoniae infection: respiratory tract, systemic, intraperitoneal chamber implant and otitis media.

\section{METHODS}

Bacterial strains and growth conditions. All strains used in these studies are derivatives of S. pneumoniae D39, an encapsulated serotype 2 strain (Tiraby et al., 1975). S. pneumoniae was routinely grown at $37{ }^{\circ} \mathrm{C}, 7 \cdot 5 \% \mathrm{CO}_{2}$ in air either in brain-heart infusion broth (BHIB; Difco Laboratories) supplemented with $5 \%$ yeast extract or on tryptic-soy agar (TSA) plates containing $5 \%$ defibrinated sheep blood (BBL). Antibiotics were used at the following concentrations: spectinomycin (spc), $500 \mu \mathrm{g} \mathrm{ml}^{-1}$; erythromycin, $0 \cdot 3 \mu \mathrm{g} \mathrm{ml}^{-1}$. The addition of $2 \mu \mathrm{M} \mathrm{MnSO}_{4}$ was essential for the survival and growth of the $p s a$ replacement mutants (Dintilhac et al., 1997).

Construction of the $S$. pneumoniae promoter-probe library and DFI conditions. The $S$. pneumoniae promoter-trap library in vector pNE1 $g f p$ has been described previously (Bartilson et al., 2000). Briefly, the library consists of 200-500 bp DNase I fragments of $S$. pneumoniae D39 DNA cloned upstream of the promoterless $g f p$ gene in $p N E 1 g f p$. Following ligation, the library was electroporated into E. coli RR1, and plasmid DNA was prepared and used to transform S. pneumoniae D39 as described (Bartilson et al., 2000). The S. pneumoniae D39 promoter-trap library was used to infect groups of three mice via intranasal instillation; as a negative control for sorting, D39 carrying the promoterless $g f p$ fusion plasmid pNE1gfp was used to infect another set of mice. In both cases infection was with $\sim 10^{6}$ organisms, which represents full coverage of the library (Bartilson et al., 2000). Both sets of mice were sacrificed $24 \mathrm{~h}$ post-infection and their lungs lavaged with $1 \mathrm{ml}$ sterile PBS. An aliquot of each recovered sample was titred to determine bacterial numbers inside the lungs; the remainder was pooled and used for sorting by flow cytometry on a FACStar machine (Becton Dickinson) equipped with an argon laser emitting at $488 \mathrm{~nm}$. The sample containing the negative control was also analysed to adjust the gate for background fluorescence. Sorted cells were collected into BHIB containing spc and this suspension was spread onto TSA plates containing $5 \%$ sheep blood and spc. After overnight growth, cells were collected with swabs into PBS and aliquots frozen at $-80^{\circ} \mathrm{C}$. After two more rounds of infection and sorting, the resulting population was plated for single colonies, and 96 individual clones were picked for PCR and sequencing of inserts. PCR was performed on an aliquot of exponential-phase cells using the Expand High Fidelity PCR System (Roche Molecular Biochemicals). Insert DNA sequences were compared with the $S$. pneumoniae genome using the BLAST algorithm at TIGR (http://www.tigr.org/) and downstream genes identified using OMIGA software (Oxford Molecular). For reasons described below, we focused on one clone that was isolated a disproportionate number of times, which we identified as $p s a$.

Construction of psa null mutants. S. pneumoniae D39 derivatives with replacement mutations in the $p s a$ genes were constructed by using plasmid pCZA342 (Hoskins et al., 1999), which encodes apramycin (for selection in E. coli) and erythromycin (for selection in $S$. pneumoniae) resistance markers. The strategy for constructing null mutants requires 
generation of a construct containing a spectinomycinresistance marker (LeBlanc et al., 1991) flanked by PCRgenerated fragments upstream and downstream of the gene to be mutated cloned into pCZA342. Plasmid pCZA342 can replicate in E. coli but not in $S$. pneumoniae so transformants are screened for an erythromycin-sensitive, spectinomycinresistant phenotype. The primers used for amplification of the cloning fragments were based on the $S$. pneumoniae unfinished genome sequence at TIGR. For $p s a A$, a 593 bp Pst I-XhoI fragment and a $636 \mathrm{bp} \mathrm{SacI-BamHI}$ fragment were ligated to the XhoI-SacI spectinomycin-resistance marker and the Pst I/ BamHI-digested pCZA342 plasmid. Oligomers used to PCR amplify the cloning fragments flanking the $p s a A$ gene were $: 5^{\prime}$ ATGCATGCCTGCAGGCTAAAGCCATGGGAATGC-3'; 5'-ATGCATGCCTCGAGGGTTCGTATTCGTGTGG-3'; 5'-ATGCATGCGAGCTCCCTATGGTGTCCCAAGTGC3'; 5'-ATGCATGCGGATCCCGTGTTGTCCAGTCCAGC-3'.

For $p s a B$, a 467 bp PstI-XhoI fragment and a 442 bp SacI$-B a m H I$ fragment were ligated to the XhoI-SacI spectinomycin-resistance marker and the PstI/BamHI-digested pCZA342 plasmid. Oligomers used to PCR amplify the cloning fragments flanking the $p s a B$ gene were: $5^{\prime}$-ATGCATGCCTGCAGGGGATTTCAGCTGTCTGTTGCC-3'; 5'-ATGCATGCCTCGAGCCGTTTGGACCAATGATGC-3'; 5'-ATGCATGCGAGCTCGGGATTGACTCTGTCAGTGAGG-3' ; 5'-ATGCATGCGGATCCGGCTCCGATAAAGAAGTCAAGG-3'.

For psaC, a 594 bp PstI-XhoI fragment and a 593 bp SacI-BamHI fragment were ligated to the XhoI-SacI spectinomycin-resistance marker and the Pst $\mathrm{I} / \mathrm{BamHI}$-digested pCZA342 plasmid. Oligomers used to PCR amplify the cloning fragments flanking the $p s a C$ gene were: $5^{\prime}$-ATGCATGCCTGCAGCCTTGAAATCGTCGGCCTAGC-3'; 5' $^{\prime}$ ATGCATGCCTCGAGGCAATCCAAAGACAATGG-3'; 5'-ATGCATGCGAGCTCGCTAAAGCCATGGGAATGC-3'; 5'-ATGCATGCGGATCCGGTTCGTATTCGTGTGG-3'.

For deleting the entire psa operon, a 467 bp Pst I-XhoI fragment and a $636 \mathrm{bp}$ SacI-BamHI fragment were ligated to the XhoI-SacI spectinomycin-resistance marker and the PstI/BamHI-digested pCZA342 plasmid. Oligomers used to PCR amplify the cloning fragments flanking the $p s a$ operon were: 5'-ATGCATGCCTGCAGGGGATTTCAGCTGTCTGTTGCC-3' ; 5'-ATGCATGCCTCGAGCCGTTTGGACCAATGATGC-3' ; 5'-ATGCATGCGAGCTCCCTATGGTGTCCCAAGTGC-3' ; 5'-ATGCATGCGGATCCCGTGTTGTCCAGTCCAGC-3'.

S. pneumoniae D39 cells were transformed with plasmid DNA of the recombinant pCZA342 derivatives that had been purified from E. coli DH12S electrocompetent cells (Gibco$\mathrm{BRL})$. The transformation of $S$. pneumoniae required bacterial cells to be in early exponential phase $\left(\mathrm{OD}_{600} 0 \cdot 05-0 \cdot 1\right)$ in BHIB containing synthetic competence-stimulating peptide CSP-1 at a final concentration of $0 \cdot 1 \mu \mathrm{g} \mathrm{m}^{-1}, 10 \mathrm{mM}$ glucose and $10 \%$ horse serum (Sigma). The bacteria were cultured on TSA with $5 \%$ sheep blood and $0.5 \mathrm{~g} \mathrm{l}^{-1} \mathrm{spc}$ (plates prepared by Becton Dickinson) in an atmosphere of $7.5 \% \mathrm{CO}_{2}$ at $37^{\circ} \mathrm{C}$. Mutants were verified by both Southern blotting and PCR amplifications using primers flanking the cloned regions (not shown). Two independent isolates were characterized for each mutant generated.
In vitro growth of psa mutants in the presence of manganese. Growth curves were produced for all four $p s a$ mutants and D39 in BHIB or BHIB supplemented with $\mathrm{MnSO}_{4}$ as follows. Strains were inoculated into $1 \mathrm{ml}$ BHIB containing $5 \%$ yeast extract, $500 \mu \mathrm{g} \mathrm{spc} \mathrm{ml} \mathrm{m}^{-1}$ (for $p s a$ mutants), $2 \mu \mathrm{M}$ $\mathrm{MnSO}_{4}$ (for psa mutants) and incubated for $6 \mathrm{~h}$ at $37^{\circ} \mathrm{C}, 7 \cdot 5 \%$ $\mathrm{CO}_{2}$. This culture was then used to inoculate $40 \mathrm{ml}$ of the same medium, which was incubated for $16 \mathrm{~h}$ at $37^{\circ} \mathrm{C}, 7 \cdot 5 \%$ $\mathrm{CO}_{2}$. Cultures were centrifuged and the cells washed in PBS, resuspended in $\mathrm{BHIB}$, and the $\mathrm{OD}_{600}$ measured. Cultures were diluted into $20 \mathrm{ml} \mathrm{BHIB} \mathrm{containing} 2 \mu \mathrm{M} \mathrm{MnSO}_{4}$, adjusted to $\mathrm{OD}_{600} 0.05$ and $1 \mathrm{ml}$ aliquots distributed into $15 \mathrm{ml}$ roundbottom culture tubes. Cultures were incubated at $37^{\circ} \mathrm{C}, 7 \cdot 5 \%$ $\mathrm{CO}_{2}$ and at $1 \mathrm{~h}$ intervals, one aliquot from each set of tubes was removed and the $\mathrm{OD}_{600}$ determined.

\section{Animal infection models}

Murine RTI. Bacterial cultures for infection were prepared by inoculating TSA $/ 5 \%$ sheep blood plates containing appropriate antibiotics or manganese with bacteria from frozen stocks. Plates were incubated overnight and growth collected with swabs into PBS. Bacterial suspensions were adjusted so that the $\mathrm{OD}_{600}$ of a $1: 10$ dilution was approximately $0 \cdot 3$. Female CD-1 mice, aged 6 weeks, were anaesthetized with isoflurane $\left(4 \%\right.$ in $\left.\mathrm{O}_{2}\right)$ and infected with $50 \mu \mathrm{l}$ bacterial suspension $\left(\sim 5 \times 10^{7}\right.$ cells $)$ by intranasal instillation. Five to ten animals were routinely infected per group. Animals were allowed to recover and given food and water ad libitum, and after $48 \mathrm{~h}$ were killed by $\mathrm{CO}_{2}$ overdose. Blood was collected by cardiac puncture and assayed for viable bacteria by agar plate count; the lungs were aseptically removed, homogenized in $1 \mathrm{ml}$ PBS in a Stomacher (Labconco), and plated onto agar for viable counts. Each experiment was performed a minimum of two times, and the data shown are composites of all experiments.

Murine systemic infection. Bacterial suspensions were prepared as described above for murine RTI. Suspensions were serially diluted 10-fold in PBS to appropriate concentrations such that mice would receive between 10 and $10^{6}$ organisms. Female CD-1 mice aged 6 weeks were given $200 \mu$ l diluted bacterial suspension by intraperitoneal injection using a 28 gauge tuberculin syringe. Six to ten mice were infected per group; mice were monitored twice daily and deaths were recorded. $\mathrm{LD}_{50}$ calculations were based on the number of survivors in each group at $22 \mathrm{~h}$ post-infection. For each mutant, a second independent isolate was used to infect groups of mice to ensure that the observed results were due to the engineered mutation and not to a second event. The secondary transformants were injected into mice at the $\mathrm{D} 39 \mathrm{LD}_{50}(40-100$ cells) and at 1000 times the $\mathrm{LD}_{50}$; in all cases the numbers of survivors corroborated the results seen with the primary transformants (data not shown).

Otitis media infections. Bacterial suspensions were prepared as described above and diluted to $\sim 10^{5}$ cells $\mathrm{ml}^{-1}$. Male Mongolian gerbils weighing 25-30 g were anaesthetized with isoflurane $\left(5 \%\right.$ in $\left.\mathrm{O}_{2}\right)$ and $30 \mu \mathrm{l}$ bacterial suspension (usually $10^{3}-10^{4}$ organisms) was injected through the bone of both the right and the left bullae using a $25 \mathrm{~g}$ needle attached to a $0.5 \mathrm{ml}$ syringe. Animals were returned to their cages and allowed food and water ad libitum and killed at $96 \mathrm{~h}$ post-infection by $\mathrm{CO}_{2}$ overdose. Upon killing, the middle ear aspirates were extracted following injection of $100 \mu \mathrm{l}$ PBS into each middle ear cavity through the tympanic membrane and withdrawal of fluid. Bacteria contained in this aspirate were enumerated following serial dilution and plating for c.f.u. on TSA $/ 5 \%$ 
sheep blood plates containing appropriate antibiotics. In addition, blood was collected by cardiac puncture and numbers of viable bacteria determined by agar plate count.

Murine intraperitoneal chamber implant model. This model requires the construction of diffusion chambers using $1 \mathrm{~cm}$ length sections of a $1 \mathrm{ml}$ syringe. Millipore MF $0.22 \mu \mathrm{m}$ pore size filters were cut to the diameter of the syringe barrels and attached to the ends of the barrels by melting on a hot plate and pressing the molten plastic onto the filter. The entire sealed chamber was then sterilized by autoclaving. Bacteria for inoculating the chambers were prepared by swabbing fresh overnight growth off a TSA $/ 5 \%$ sheep blood plate into PBS and diluting to the desired cell density. Bacteria were injected into the chamber with a needle and the injection site was sealed with a heated glass rod. In some experiments, $20 \mu \mathrm{M}$ $\mathrm{MnSO}_{4}$ was added to the chambers. Mice (female CD-1, age 7-8 weeks) were anaesthetized with 3-4\% isoflurane, and the abdomen shaved and swabbed with betadine. A small longitudinal incision $(2-3 \mathrm{~cm})$ was made in the abdomen and one to four chambers placed into the peritoneal cavity. The abdominal wall was then sutured closed using 3/0 gauge silk sutures and the skin stapled closed. Animals were allowed to recover and given food and water ad libitum. At a given time point mice were killed by $\mathrm{CO}_{2}$ overdose and chambers were removed aseptically. Bacteria were removed from the chamber using a syringe and viable bacterial counts determined by plating serial dilutions onto TSA plates containing $5 \%$ sheep blood with antibiotic selection, where appropriate.

Induction of psa-gfp fusion in vivo. For analysis of $p s a-g f p$ fusion expression in vivo, D39 cells carrying the $p s a-g f p$ fusion plasmid isolated by multiple rounds of RTI followed by sorting were grown in BHIB containing $5 \%$ yeast extract and spc to exponential phase. Cells were washed in PBS and an aliquot was analysed for fluorescence on a FACS Caliber machine (Becton Dickinson) with an argon laser emitting at $488 \mathrm{~nm}$. The washed cells were also used to load multiple diffusion chambers for peritoneal implant of a mouse. As a negative control, the D39 strain carrying the promoterless $g f p$ fusion plasmid was also loaded into chambers and implanted. After $24 \mathrm{~h}$ the chambers were removed and bacteria harvested from the chambers. Recovered bacteria were analysed on the FACS Caliber machine as described above and their fluorescence levels compared to those prior to implant.

\section{RESULTS}

\section{Enrichment for promoter clones expressed during infection, and identification of a psaB-gfp fusion clone}

The basic approach for DFI technology has been described in detail elsewhere (Bartilson et al., 2000; Valdivia \& Falkow, 1997). We have adapted this technology for S. pneumoniae, constructing a random promoter-probe library that relies on GFP expression and FACS following growth of the library under inducing conditions (Bartilson et al., 2000; Marra et al., 2002). Sorting allows for the collection of clones based on their GFP expression, presumably due to a randomly cloned promoter element upstream of the $g f p$ gene that responds to the inducing conditions. Of particular interest to us were genes induced during in vivo infection and we focused our efforts on murine RTI. Following three rounds of RTI and sorting for induced clones, inserts from 100-200 individual randomly picked clones
Table 1. Induction of $p s a B-g f p$ fusion in intraperitoneally implanted chambers harvested at $22 \mathrm{~h}$

Bacteria were prepared as described in Methods and loaded into chambers.

\begin{tabular}{|cccc|}
\hline Experiment & $\begin{array}{c}\text { m.c.f. inoculum } \\
\text { (uninduced) }\end{array}$ & $\begin{array}{c}\text { m.c.f. harvest } \dagger \\
\text { (induced) }\end{array}$ & Ratio $\neq$ \\
\hline 1 & $12 \cdot 5$ & $156 \cdot 0$ & $12 \cdot 5$ \\
(12 replicates) & & $181 \cdot 8$ & $14 \cdot 5$ \\
& & $173 \cdot 7$ & $13 \cdot 9$ \\
& & $229 \cdot 6$ & $18 \cdot 3$ \\
& & $215 \cdot 1$ & $17 \cdot 2$ \\
& $190 \cdot 9$ & $15 \cdot 3$ \\
& & $137 \cdot 6$ & $10 \cdot 9$ \\
& & $118 \cdot 2$ & $9 \cdot 4$ \\
& & $167 \cdot 9$ & $13 \cdot 4$ \\
& & $156 \cdot 3$ & $12 \cdot 5$ \\
& & $181 \cdot 9$ & $14 \cdot 5$ \\
& & $129 \cdot 5$ & $10 \cdot 4$ \\
& & $222 \cdot 9$ & $9 \cdot 1$ \\
& & $184 \cdot 3$ & $7 \cdot 6$ \\
& & $220 \cdot 9$ & $9 \cdot 1$ \\
\hline
\end{tabular}

*Fluorescence of input bacteria.

† Bacteria were recovered from chambers after having been implanted for $22 \mathrm{~h}$ and the m.c.f. determined.

‡ The ratio of induced to uninduced m.c.f. values.

were PCR amplified and sequenced. In several such experiments, the $p s a-g f p$ fusion clone was isolated more than once and so we chose it for further study.

\section{Induction of psaB-gfp fusion in vivo}

To confirm that the $p s a$ operon is induced in vivo, as indicated by DFI, we used D39 carrying the $p s a-g f p$ fusion to load diffusion chambers. The inoculum was analysed by flow cytometry (the 'uninduced' condition) and multiple chambers were implanted into the peritoneal cavities of mice. Chambers were recovered at $22 \mathrm{~h}$ post-infection and the bacteria harvested from the chambers were again analysed by flow cytometry ('induced' condition). The ratio between induced and uninduced mean channel fluorescence (m.c.f.) values is shown in Table 1. Two independent experiments indicated that the $p s a$ promoter was consistently induced approximately 10 -fold in vivo. The negative control, strain D39 carrying the promoterless $g f p$ vector, showed no induction in vivo (not shown).

\section{Characterization of psa null mutants in vitro}

We constructed deletion mutants of the individual $p s a$ genes and the entire $p s a$ operon. To study the trace metal requirements for growth in a $p s a$ mutant background, the four $p s a$ mutants and the wild-type parent strain were grown in BHIB or BHIB containing manganese, manganese and iron, manganese and zinc, or all 


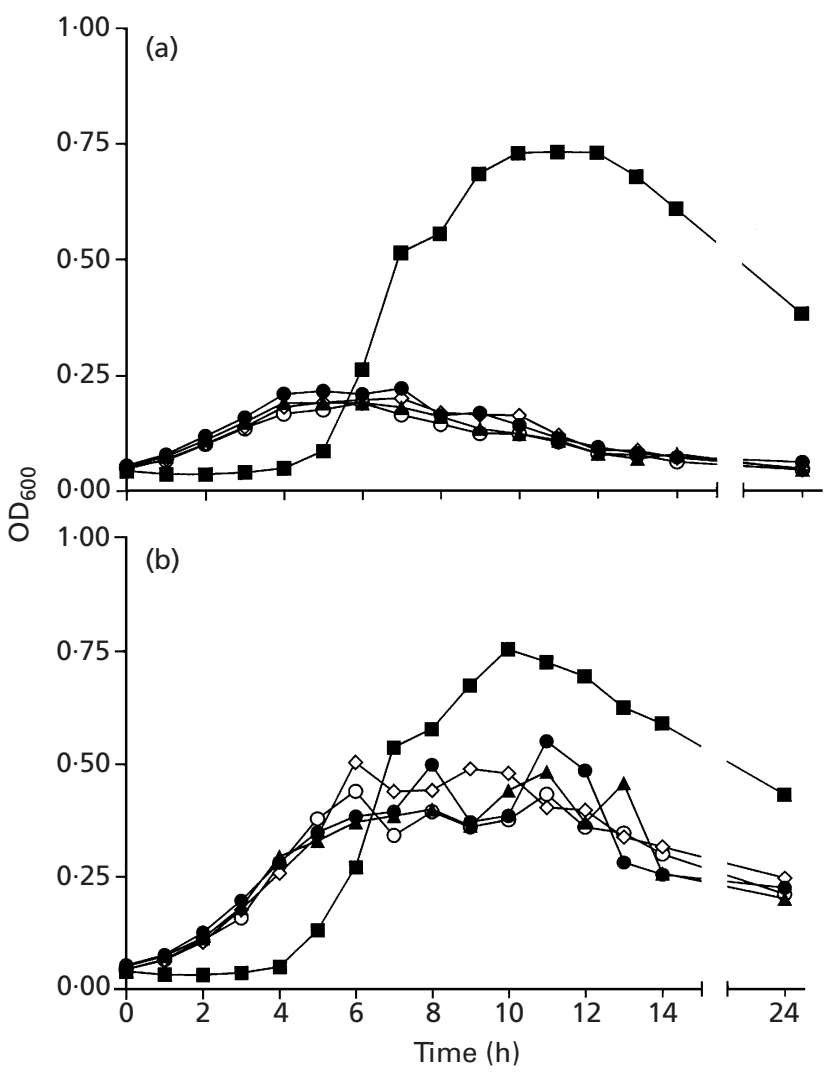

Fig. 1. Time course of bacterial growth in $\mathrm{BHIB}+\mathrm{MnSO}_{4}$. Bacterial strains were grown as described in Methods and inoculated into either BHIB (a) or $\mathrm{BHIB}+2 \mu \mathrm{M} \mathrm{MnSO}_{4}$ (b). $\mathbf{\square}$, $\mathrm{D} 39 ; \boldsymbol{\Lambda}, p s a A ; 0, p s a B ; \diamond, p s a C ; \bigcirc$, psaBCA.

three metals at concentrations of $100 \mu \mathrm{M}$ each. Only manganese stimulated growth of the $p s a$ mutants in BHIB (not shown), whereas strain D39 was able to grow to high densities in all five media. Addition of iron or zinc to BHIB did not restore growth to any psa mutants (not shown), and BHIB containing manganese plus the other metals had a similar effect to manganese alone.

We next looked at the growth kinetics of D39 and the four psa mutants in BHIB compared to BHIB supplemented with manganese (Fig. 1). D39 was able to grow in unsupplemented BHIB (Fig. 1a), whereas the four $p s a$ mutants showed slight growth between 2 and $4 \mathrm{~h}$ but only reached approximately $30 \%$ of the cell density achieved by D39. In contrast, when manganese was added all four $p s a$ mutants were capable of growth to approximately $67 \%$ of wild-type levels (Fig. 1b). Also, the psa mutants have a much shorter lag time than D39 in this medium. Higher concentrations of manganese failed to further increase growth of the mutants.

\section{Characterization of psa mutants in vivo}

RTI model. All three $p s a$ mutants, as well as the mutant carrying the deletion of the entire $p s a$ operon, were used to infect mice by intranasal instillation to establish a
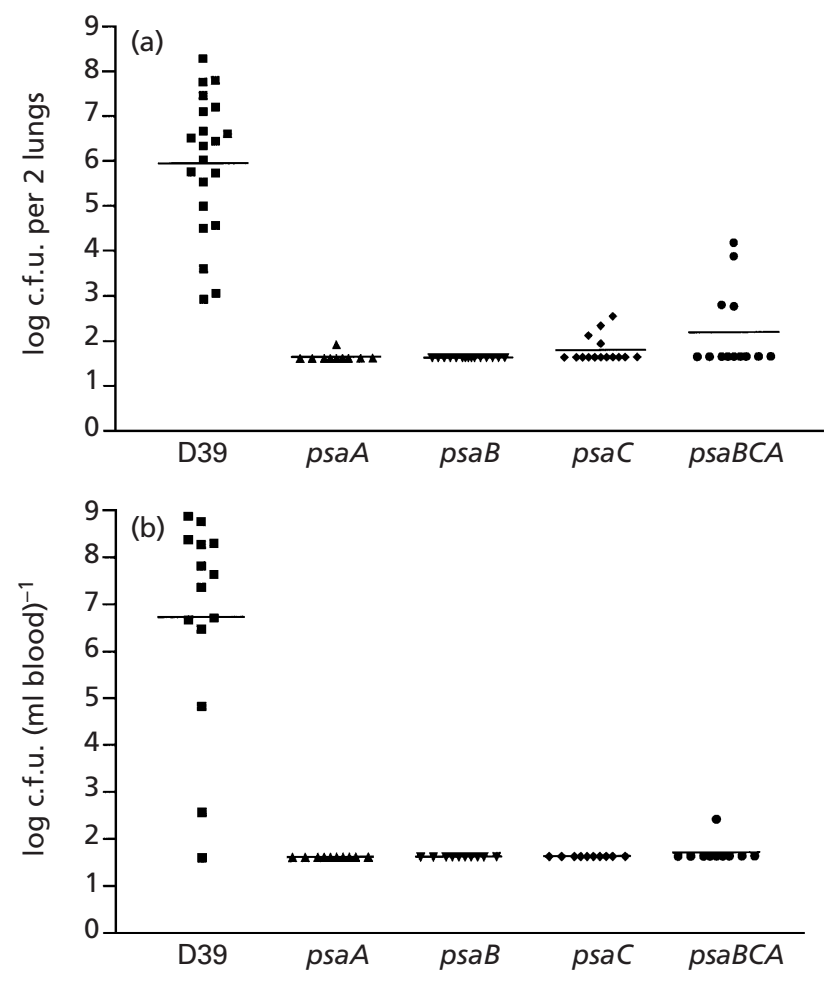

Fig. 2. S. pneumoniae D39 and psa mutants in $48 \mathrm{~h} \mathrm{RTI}$ in mice. Mice were infected as described and bacterial counts in the lungs (a) and blood (b) are shown. Horizontal bars represent geometric means. Graphs shown are composites of four experiments. The limit of detection is 40 organisms.

RTI. Lung homogenates were titred for bacterial counts $48 \mathrm{~h}$ after infection. The results are shown in Fig. 2(a). The $p s a A$ and $p s a B$ mutants were unable to colonize the lungs, with homogenates yielding bacterial numbers at or below the limit of detection. In a minority of mice infected with the $p s a C$ or the $p s a B C A$ mutants, small numbers of bacteria were found in the lungs. In contrast, the mean number of bacteria found in the lungs following infection with the wild-type D39 strain was $10^{6}$. Since D39 is known to cause systemic as well as RTI following intranasal inoculation, bacteria in the blood were also enumerated. As expected, the D39 strain was found at very high titres in the blood of intranasally infected mice, often at $10^{7}-10^{8}$ c.f.u. $\mathrm{ml}^{-1}$. However, none of the psa mutant bacteria were found in the blood after 48 h (Fig. 2b).

Otitis media infection. In a model of otitis media infection, Mongolian gerbils were infected with one of the four $p s a$ mutants or the wild-type strain and middle ear aspirates were collected. As in the RTI model, in this model strain D39 has the ability to disseminate systemically, so blood was also collected from infected animals. Over the 4 days of infection, parental strain D39 can survive and multiply approximately 100000 -fold in the middle ear, and dissemination to the blood is evident, as shown in Fig. 3. In contrast, no bacteria from any of the $p s a$ mutants were recovered from gerbil middle ear cavities 

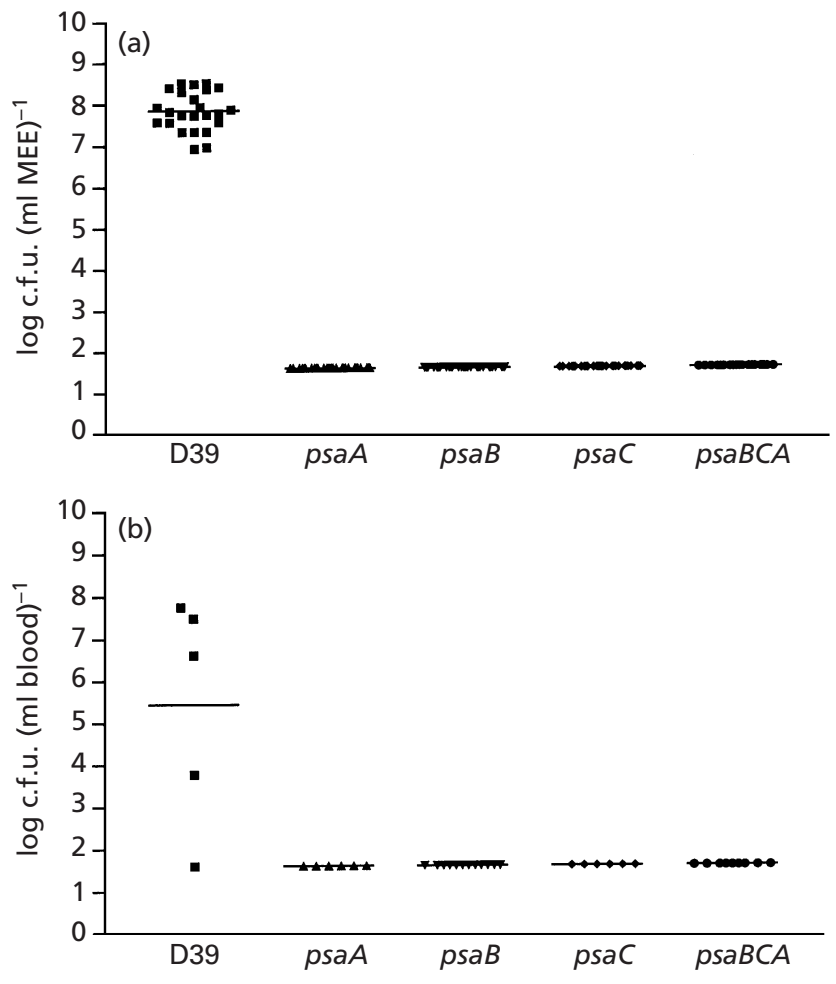

Fig. 3. S. pneumoniae psa mutants in 4 day otitis media infection in gerbils. Gerbils were infected as described in Methods and bacterial counts in middle ear exudate (MEE) (a) and blood (b) are shown. Horizontal bars represent geometric means. (a) is a composite of six experiments, (b) is a composite of two experiments. The limit of detection is 40 organisms.

Table 2. $L D_{50}$ values (c.f.u.) for psa mutants following $24 \mathrm{~h}$ systemic infection

Mice were infected by intraperitoneal injection as described in Methods. $\mathrm{LD}_{50}$ values were calculated based on the number of surviving animals at $24 \mathrm{~h}$ post-infection.

\begin{tabular}{|lcc|}
\hline Strain & Experiment 1 & Experiment 2 \\
\hline D39 & 38 & 87 \\
$\Delta p s a B$ & $>6.5 \times 10^{5}$ & $>7 \cdot 6 \times 10^{5}$ \\
$\Delta p s a C$ & $>8.8 \times 10^{5}$ & $>1 \cdot 1 \times 10^{6}$ \\
$\Delta p s a A$ & $>1 \times 10^{6}$ & $>8 \cdot 1 \times 10^{5}$ \\
$\Delta p s a B C A$ & $>1.7 \times 10^{6}$ & $>1 \cdot 2 \times 10^{6}$ \\
\hline
\end{tabular}

(Fig. 3a). Blood collected from the psa mutant-infected mice 4 days post-infection yielded no detectable bacteria (Fig. 3b). With the idea of rescuing the psa mutants, in two experiments, a 50- or 500-fold excess of $\mathrm{MnSO}_{4}$ was concomitantly injected into the middle ear cavities, yet still no bacteria were recovered (data not shown).

Systemic infection. To determine the effects of the $p s a$ mutations on the ability of this organism to cause systemic infection and death, groups of mice were

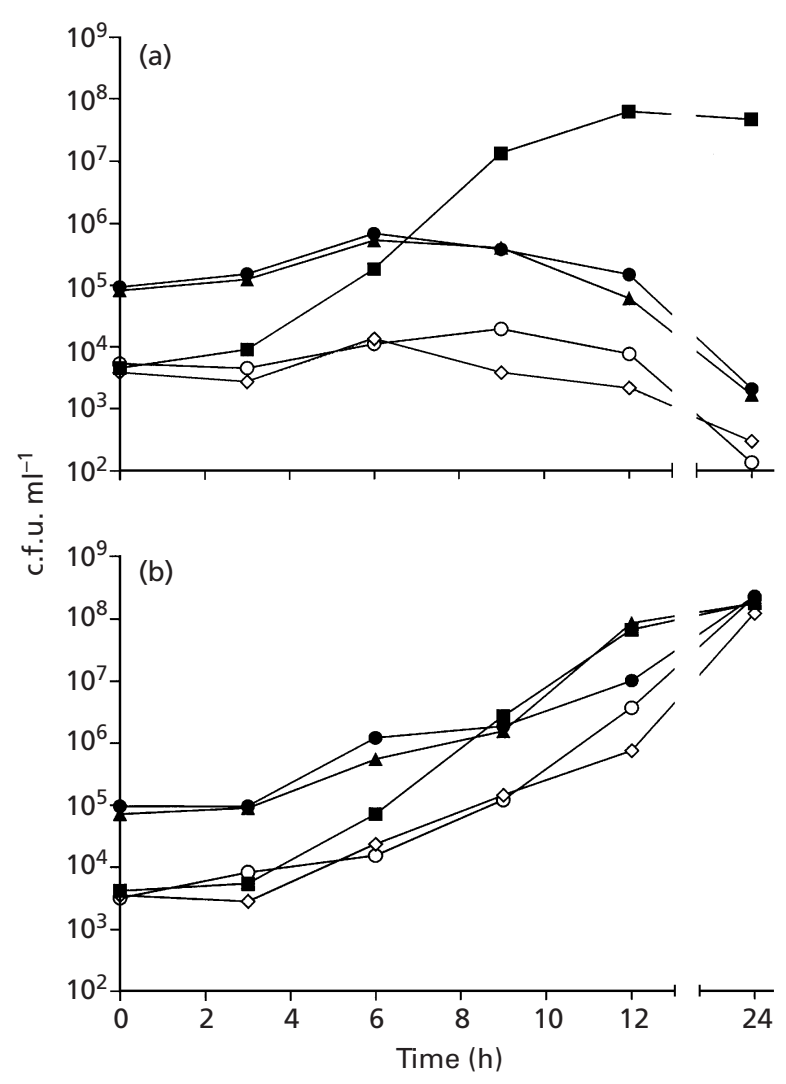

Fig. 4. Time course of $S$. pneumoniae D39 and psa mutants in intraperitoneal chamber implant. Chambers were prepared and implanted as described in Methods. At the indicated time points, bacterial growth within the chambers was quantitated. (a) Bacterial growth within implanted chambers containing PBS. (b) Bacterial growth within implanted chambers containing $\mathrm{PBS}+20 \mu \mathrm{M} \mathrm{MnSO}_{4}$., $\mathrm{D} 39 ; \boldsymbol{\Delta}$, psaA;, psaB; $\diamond, p s a C ; 0$, psaBCA.

injected intraperitoneally. with different doses of bacteria and the $\mathrm{LD}_{50}$ for each strain was calculated at $24 \mathrm{~h}$ post-infection. Table 2 shows that the $\mathrm{LD}_{50}$ values for the $p s a$ mutants are approximately 10000 -fold greater than for the parental strain. In addition, mice infected with any of the $p s a$ mutants remained alive for at least 1 week after infection without showing any signs of illness, whereas all mice infected with D39 at any dose died within 3 days.

Intraperitoneal chamber implant. To help address the question of whether the decrease in virulence of the $p s a$ mutants was due to increased susceptibility to host defences or to a nutritional requirement, we loaded diffusion chambers with either D39 or psa mutants in PBS, implanted them into the peritoneal cavity of mice and measured their ability to multiply over a $24 \mathrm{~h}$ period. Strain D39 was able to multiply approximately 1000 -fold in this period, reaching levels of $10^{8}$ c.f.u. $\mathrm{ml}^{-1}$. Mutants $p s a A, p s a B, p s a C$ and the triple mutant all lost viability over $24 \mathrm{~h}$ (Fig. $4 \mathrm{a}$ ). We wanted to see whether the addition of manganese to the chambers would restore growth to these mutants. As shown in Fig. 4(b), all four mutant strains grew to wild-type levels when the 
chambers were supplemented with $20 \mu \mathrm{M} \quad \mathrm{MnSO}_{4}$. Thus, the attenuated growth phenotype of the $p s a$ mutants in the diffusion chambers can be corrected by the addition of excess manganese.

\section{DISCUSSION}

The psa genes of $S$. pneumoniae have been described previously as encoding a manganese uptake system for this organism (Dintilhac et al., 1997). Insertion mutations in $p s a B, p s a C$ or $p s a A$ have been demonstrated to have pleiotropic effects, including a growth requirement for added manganese and reduced tranformability (Dintilhac et al., 1997), penicillin tolerance, decreased adhesion and attenuation in vivo (Novak et al., 1998), although several of these findings have been disputed (Claverys et al., 1999). We have focused on and extended the in vivo studies by constructing more stable replacement mutants and assessing their virulence in different animal models of infection.

We first became interested in the psa locus during a screen for S. pneumoniae promoters induced during infection. Using DFI technology in the murine RTI model, the promoter upstream of $p s a B$ was isolated numerous times, indicating that it is strongly induced in vivo. Indeed, subsequent experiments using the murine intraperitoneal chamber implant model have corroborated this observation, showing that the $p s a$ promoter is induced approximately ten-fold in vivo.

The effects of these mutations were assessed in four relevant animal infection models, each of which presumably presents a different environment and challenge to the bacteria. In a systemic infection model, the $\mathrm{LD}_{50}$ of all four $p s a$ mutants were approximately $10^{4}$-fold higher than for D39. Mice infected with even higher numbers of $p s a$ mutants survived the study, whereas the D39-infected group had very few survivors at 48 h postinfection, even at the lowest dose. It is worth noting that even when injected with over $10^{6}$ organisms, mice infected with the $p s a$ mutants did not show any signs of illness and did not succumb to infection for at least 7 days. This result is significant in that it demonstrates that the $p s a$ system is essential for the survival of this organism in vivo and that the likelihood of a suppressor mutation arising is very low, even under the strong selective pressure that might be expected to be present in vivo.

These mutants are also unable to grow in the relatively protected environment of an intraperitoneally implanted chamber. The wild-type D39 strain can multiply to large numbers, yet very few mutant bacteria were found in the chambers upon harvest. Indeed, it appears that these four mutants die within the chambers, since the numbers of bacteria used to inoculate the chambers were not recovered only $24 \mathrm{~h}$ after implant. However, when manganese is added to the chambers prior to implant, the mutants attain wild-type levels. These results together suggest that the mutations in $p s a$ are lethal in the absence of accessible manganese, as these mutants seem to lose viability by 24 h even when protected from host cells inside the chambers.

One interesting observation from this experiment is that in the intraperitoneal chambers, D39 was able to grow to high cell densities without lysing, and the psa mutants grown in the presence of manganese showed similar behaviour. This result may indicate that autolysis is more a feature of in vitro growth and does not occur so readily in vivo. Alternatively, autolysin-mediated lysis may be induced in vivo by a host factor that is not accessible to bacteria inside the chambers.

The four $p s a$ mutants also had varied effects on virulence in the murine RTI model. $p s a B$ and $p s a A$ were completely unable to colonize lungs following intranasal inoculation: no bacteria were recovered from any mice infected with these strains. A subset of mice infected with the $p s a C$ and the $p s a B C A$ mutants, however, showed very low bacterial counts (in the order of $<100$ c.f.u. per pair of lungs). In both cases the numbers of mice with lung counts at $48 \mathrm{~h}$ were approximately $25 \%$ of the total infected. It is not clear whether these counts are due to a low level of bacterial replication or to incomplete clearance by the host; it is likely to be the latter as these mutants are deletions and therefore cannot revert. The $p s a B C A$ result suggests that the deletion of all three genes leads to less severe attenuation than deletion of either gene alone, and that perhaps the absence of one component of the psa system is more deleterious than deletion of the entire system, perhaps by interfering with another uptake pathway(s).

It has been our experience that strain D39 is able to disseminate into the bloodstream following RTI in mice where the lung counts have reached $10^{4}$. This bloodstream dissemination following RTI as well as otitis media infection distinguishes S. pneumoniae from other pneumonia-causing organisms and allows this organism to cause more serious, life-threatening infections. The $p s a$ mutants do not disseminate into the bloodstream of infected mice, though it is not known whether this is due to their inability to achieve high enough levels in the lungs or whether they are more susceptible to host defences once they do get into the blood. The results of our systemic infections would suggest that both factors are at play. The nutritional defect of the $p s a$ mutants may render them less able to overcome host defences.

None of the $p s a$ mutants showed any growth in the gerbil otitis media infection, whereas the wild-type parent D39 was able to multiply $10^{3}-10^{4}$-fold within 4 days. Not surprisingly, $p s a$ mutants do not disseminate into the bloodstream in this model, as does D39. It is interesting to note that it has been reported that alveolar and middle ear macrophages can only weakly phagocytose S. pneumoniae in vitro (Bakaletz et al., 1987; Nibbering et al., 1989), lending support to the idea that these mutants die in the absence of manganese, instead of being more easily phagocytosed.

The importance of trace minerals for bacterial growth in vivo is well known (Jakubovics \& Jenkinson, 2001), so 
it is logical that any of the $p s a$ mutations would have an adverse effect on virulence, as was predicted by Dintilhac et al. (1997). It is clear that the $p s a$ mutants are unable to grow in vitro on even rich medium in the absence of added manganese, therefore these genes are, under most conditions, essential in this organism. In experiments where manganese and other trace metals were added to the medium, the $p s a$ mutants could grow but were unable to reach wild-type levels of cell density, even at high manganese concentrations (Dintilhac et al., 1997). This result suggests that the psa mutants can take up small amounts of manganese, but for full growth an active uptake system $(p s a)$ is necessary.

Previous groups have constructed different $p s a$ mutants and studied their virulence. However, these earlier mutants were not complete replacement mutants as we have generated here, but were insertion/duplication mutants. Under selective pressure such mutants are able to revert to wild-type; indeed it has been demonstrated that there is significant in vivo reversion in a systemic model of infection when large numbers of such mutant bacteria were injected (Berry \& Paton, 1996). Our in vivo analyses of the $p s a$ genes clearly demonstrate the importance of this system for the pathogenesis of $S$. pneumoniae, and indicate that the requirement for manganese is essential for virulence as well as survival. The inability of these mutants to grow in various sites in vivo addresses the issue of nutrient accessibility at these sites.

\section{ACKNOWLEDGEMENTS}

We would like to thank Patricia Lekas, Dixie Polakoff, Christopher A. Zook, Roman Moniz and Edwin Apilado for technical assistance, and Stanley Falkow, Sun Ho, Thalia Nicas and Don LeBlanc for helpful discussions.

\section{REFERENCES}

Bakaletz, L. O., DeMaria, T. F. \& Lim, D. J. (1987). Phagocytosis and killing of bacteria by middle ear macrophages. Arch Otolaryngol Head Neck Surg 113, 138-144.

Bartilson, M., Marra, A., Christine, J., Asundi, J. S., Schneider, W. P. \& Hromockyj, A. E. (2000). Differential fluorescence induction reveals Streptococcus pneumoniae loci regulated by competence peptide. Mol Microbiol 39, 126-135.

Berry, A. M. \& Paton, J. C. (1996). Sequence heterogeneity of PsaA, a 37-kilodalton putative adhesin essential for virulence of Streptococcus pneumoniae. Infect Immun 64, 5255-5262.

Brooks-Walter, A., Briles, D. E. \& Hollingshead, S. K. (1999). The pspC gene of Streptococcus pneumoniae encodes a polymorphic protein, PspC, which elicits cross-reactive antibodies to PspA and provides immunity to pneumococcal bacteremia. Infect Immun 67, 6533-6542.

Burnette-Curley, D., Wells, V., Viscount, H., Munro, C. L., Fenno, J. C., Fives-Taylor, P. \& Macrina, F. (1995). FimA, a major virulence factor associated with Streptococcus parasanguis endocarditis. Infect Immun 63, 4669-4674.

Canvin, J. R., Marvin, A. P., Sivakumaran, M., Paton, J. C., Boulnois, G. J., Andrew, P. W. \& Mitchell, T. J. (1995). The role of pneumolysin and autolysin in the pathology of pneumonia and septicemia in mice infected with a type 2 pneumococcus. J Infect Dis 172, 119-123.

Claverys, J.-P., Granadel, C., Berry, A. M. \& Paton, J. C. (1999). Penicillin tolerance in Streptococcus pneumoniae, autolysis and the Psa ATP-binding cassette (ABC) manganese permease. Mol Microbiol 32, 881-883.

Dintilhac, A. \& Claverys, J.-P. (1997). The adc locus, which affects competence for genetic transformation in Streptococcus pneumoniae, encodes an $\mathrm{ABC}$ transporter with a putative lipoprotein homologous to a family of streptococcal adhesins. Res Microbiol 148, 119-131.

Dintilhac, A., Alloing, G., Granadel, C. \& Claverys, J.-P. (1997). Competence and virulence of Streptococcus pneumoniae: Adc and PsaA mutants exhibit a requirement for $\mathrm{Zn}$ and $\mathrm{Mn}$ resulting from inactivation of putative ABC metal permeases. Mol Microbiol 25, 727-739.

Ganeshkumar, N., Hannam, P., Kolenbrander, P. \& McBride, B. (1991). Nucleotide sequence of a gene coding for a saliva-binding protein ( $\mathrm{SsaB}$ ) from Streptococcus sanguis 12 and possible role of the protein in coaggregation with actinomyces. Infect Immun 59, 1093-1099.

Hoskins, J., Matsushima, P., Mullen, D. L., Tang, J., Zhao, G., Meier, T. I., Nicas, T. I. \& Jaskunas, S. R. (1999). Gene disruption studies of penicillin-binding proteins $1 \mathrm{a}, 1 \mathrm{~b}$, and $2 \mathrm{a}$ in Streptococcus pneumoniae. J Bacteriol 181, 6552-6555.

Jakubovics, N. S. \& Jenkinson, H. F. (2001). Out of the iron age: new insights into the critical role of manganese homeostasis in bacteria. Microbiology 147, 1709-1718.

Kalin, M. (1998). Pneumococcal serotypes and their clinical relevance. Thorax 153, 159-162.

Kolenbrander, P. E., Andersen, R. N., Baker, R. A. \& Jenkinson, H. F. (1998). The adhesion-associated sca operon in Streptococcus gordonii encodes an inducible high-affinity $\mathrm{ABC}$ transporter for $\mathrm{Mn}^{2+}$ uptake. J Bacteriol 180, 290-295.

LeBlanc, D., Lee, L. \& Inamine, J. (1991). Cloning and nucleotide base sequence analysis of a spectinomycin adenyltransferase aad(9) determinant from Enterococcus faecalis. Antimicrob Agents Chemother 35, 1804-1810.

Marra, A., Asundi, J., Bartilson, M. \& 7 other authors (2002). Differential fluorescence induction analysis of Streptococcus pneumoniae identifies genes involved in pathogenesis. Infect Immun 70, 1422-1433.

Mitchell, T. J. \& Andrew, P. W. (1997). Biological properties of pneumolysin. Microb Drug Resist 3, 19-26.

Nibbering, P. H., van den Barselaar, M. T., van de Gevel, J. S., Leijh, P. C. J. \& van Furth, R. (1989). Deficient intracellular killing of bacteria by murine alveolar macrophages. Am J Respir Cell Mol Biol 1, 417-422.

Novak, R., Braun, J. S., Charpentier, E. \& Tuomanen, E. (1998). Penicillin tolerance genes of Streptococcus pneumoniae: the ABCtype manganese permease complex Psa. Mol Microbiol 29, 1285-1296.

Ogunniyi, A. D., Folland, R. L., Briles, D. E., Hollingshead, S. K. \& Paton, J. C. (2000). Immunization of mice with combinations of pneumococcal virulence proteins elicits enhanced protection against challenge with Streptococcus pneumoniae. Infect Immun 68, 3028-3033.

Paton, J. C. (1996). The contribution of pneumolysin to the pathogenicity of Streptococcus pneumoniae. Trends Microbiol 4, 103-106.

Paton, J. C., Andrew, P. W., Boulnois, G. J. \& Mitchell, T. J. (1993). Molecular analysis of the pathogenicity of Streptococcus pneu- 
moniae: the role of pneumococcal proteins. Annu Rev Microbiol 47, 89-115.

Paton, J. C., Berry, A. M. \& Lock, R. A. (1997). Molecular analysis of putative pneumococcal virulence proteins. Microb Drug Resist 3, 1-10.

Reynolds, H. Y. (1999). Defense mechanisms against infections. Curr Opin Pulm Med 5, 136-142.

Sampson, J. S., O'Connor, S. P., Stinson, A. R., Tharpe, J. A. \& Russell, H. (1994). Cloning and nucleotide sequence analysis of psaA, the Streptococcus pneumoniae gene encoding a 37kilodalton protein homologous to previously reported Streptococcus sp. adhesins. Infect Immun 62, 319-324.

Srivastava, N., Zeiler, J. L., Smithson, S. L., Carlone, G. M., Ades, E. W., Sampson, J. S., Johnson, S. E., Kieber-Emmons, T. \& Westerink, M. A. J. (2000). Selection of an immunogenic and protective epitope of the PsaA protein of Streptococcus pneumoniae using a phage display library. Hybridoma 19, 23-31.

Talkington, D. F., Crimmins, D. L., Voellinger, D. C., Yother, J. \& Briles, D. E. (1991). A 43-kilodalton pneumococcal surface protein, PspA : isolation, protective abilities and structural analysis of the amino terminal sequence. Infect Immun 59, 1285-1289.

Talkington, D. F., Brown, B. G., Tharpe, J. A., Koenig, A. \& Russell, H. (1996). Protection of mice against fatal pneumococcal challenge by immunization with pneumococcal surface adhesin A (PsaA). Microb Pathog 21, 17-22.

Tiraby, G., Fox, M. S. \& Bernheimer, H. (1975). Marker discrimination in deoxyribonucleic acid-mediated transformation of various pneumococcus strains. J Bacteriol 121, 608-618.

Valdivia, R. H. \& Falkow, S. (1996). Bacterial genetics by flow cytometry: rapid isolation of Salmonella typhimurium acidinducible promoters by differential fluorescence induction. Mol Microbiol 22, 367-378.

Valdivia, R. H. \& Falkow, S. (1997). Fluorescence-based isolation of bacterial genes expressed within host cells. Science 277, 2007-2011.

Watson, D. A., Musher, D. M. \& Verhoef, J. (1995). Pneumococcal virulence factors and host immune responses to them. Eur J Clin Microbiol Infect Dis 14, 479-490.

Zysk, G., Schneider-Wald, B. K., Hwang, J. H., Bejo, L., Kim, K. S., Mitchell, T. J., Hakenbeck, R. \& Heinz, H.-P. (2001). Pneumolysin is the main inducer of cytotoxicity to brain microvascular endothelial cells caused by Streptococcus pneumoniae. Infect Immun 69, 845-852.

Received 2 November 2001; revised 7 January 2002; accepted 16 January 2002. 